\title{
Quantitative genetics focus issue
}

\section{Heredity (2005) 94, 273-274. doi:10.1038/sj.hdy.6800646}

In this issue, we are highlighting papers representing recent innovations in quantitative genetics. They reflect the extension of the discipline, on both experimental and theoretical fronts. This overview provides a brief introduction to their promise; it is aimed at the more general Heredity reader, who might otherwise be wary of quantitative genetics papers.

One paper, by Sved and Chen, describes the exploitation of recombinant inbred lines (RILs). This term refers to a series of lines descended from a cross and established at the F2 generation or later. As a line has been inbred, its genome will be homozygous for blocks descended from one parent or the other. Different lines will have different segments of the genome fixed for a different parental origin. Among other benefits, this design allows the use of large sample size and repeated experiments to assess the phenotypic effects of the genetic differences that were segregating in the initial cross.

Sved and Chen follow up their observations on the results of crossing RILs of the model laboratory plant, Arabidopsis thaliana, back to the original parental lines. They were surprised at the superior performance of many of these crosses. By scoring genetic markers, they were able to determine which segments of the genome had been made heterozygous by the crosses, and have begun to dissect out the relative importance of heterozygosity across the genome as a whole, heterozygosity at particular loci and interactions between loci (epistasis).

Investigating the nature and importance of interactions among loci has proved difficult, especially for species that are less tractable for designed crosses than model organisms such as Arabidopsis. As an alternative, a theoretical paper by Mao and Xu shows that it is possible to make efficient use of information from arbitrary complicated pedigrees with incomplete marker information. They use the genotypes at genetic markers to assess the parts of the genome that are identical by descent within pedigree. The analysis extends to different loci and different individuals within the pedigree: so, for example, it deals with the probability that two different individuals in the pedigree share a haplotype extending between two loci. Their method takes account the suite of possible patterns of identity and their relative plausibility, whereas previous approaches had attributed an average effect to each locus. Their more appropriate treatment makes it possible to evaluate efficiently the evidence that multiple loci affect a particular trait, and, in particular, whether there are interactions between the effects of each locus.

Leamy et al's quantitative analysis of the genetic variation that underlies variation in fluctuating asymmetry (FA; nondirectional differences between left and right sides of bilateral characters) in the size and shape of mouse molars illustrates just how important taking account of such epistatic effects can be. Their whole genome scan only turned up two quantitative trait loci (QTLs) that significantly affected FA in molar shape but showed that about $20 \%$ of the total variance for FA of both size and shape was attributable to epistatic interactions among loci.

For some species, it is not plausible to carry out the sort of extensive crosses possible in model organisms, but it is possible to obtain sizable F2 families. These will typically have been obtained from a cross between diverged lines. If genetic markers and phenotypic traits are recorded for the family, it is possible to infer the genomic location of loci that affect the traits from the correlation between phenotype and marker genotype. Many previous analyses have been based on the premise that the grandparental generation was homozygous at the loci with phenotypic effects (the QTLs).

To counter this, Hayashi has developed a method that allows for some QTLs having been homozygous and others heterozygous in the grandparental generation. This is likely to be relevant in cases where the grandparental generation was a cross between outbred lines, which are more likely to comprise heterozygous individuals. This more sophisticated approach can improve the efficiency of the analysis. Furthermore, in some cases, the members of the family will still be alive at the end of the experiment and may be used for future breeding. As the method can identify which individuals were likely to be homozygous at which QTL, it can be used to plan the selection of individuals for a breeding program.

Further away from direct application is the theoretical paper by Hu. Here, genotype data from a single generation can be used to estimate the correlation in identity-by-descent among two or three loci. For example, if two loci are closely linked, then the identity-bydescent of two homologous chromosomes at one locus will imply identity at the other unless there has been recombination during their common history. The map distance over which such correlations are observed will be affected by processes such as hitchhiking of linked loci as an advantageous alleles spreads, or conversely background selection removing linked deleterious alleles or, additionally, simply by the magnitude of genetic drift. These patterns are emerging from large mapping projects, but the methods for characterising them are still in their infancy.

More direct evidence of the effects of selection comes from the distortion of the expected Mendelian transmission ratios of genetic markers. Most often, this will indicate the action of selection on linked loci rather than the markers themselves. The paper by Luo et al extends existing methods to estimate selection and dominance, and provides significance tests. Even more ingeniously, they show how the analysis of fitness can be subjected to existing techniques developed for QTL analysis. They consider an imaginary trait, 'liability', invisible to the investigator but visible to nature. If an individual's trait value exceeds a threshold, then the individual survives. This approach allows several advances: the partitioning 
of selection into additive and dominance effects, and the ability to remove the effects of non-genetic cofactors from the analysis.

In several of these papers, the discipline of quantitative genetics is extending its scope into new areas of evolutionary investigation. In part, this reflects technological advances: the burgeoning information from genome projects has provided markers and genetic maps, and the advances in computer technology have allowed more complex analyses. And of course, the ingenuity of the authors marks a further vital contribution.

RA Nichols Editor E-mail: r.a.nichols@qmul.ac.uk 